Sri Lankan J. Biol. 2021, 6 (2): 20-31

DOI: http://doi.org/10.4038/sljb.v6i2.81

https://orcid.org/0000-0002-5086-0215

Research Article

Open Access

\title{
Divergence of plastid 2-oxoglutarate "only" transporters away from general transporters by using a cysteine-rich architecture.
}

Gunawardana, D.*

Research Council, University of Sri Jayewardenepura, Gangodawila, Sri Lanka.

\begin{abstract}
The common carbon and nitrogen currency, 2-oxoglutarate, could become a valuable resource for nitrogen assimilation and carbon centered biochemical fates. Here in this in silico study, a myriad of factors was used, namely phylogeny, sequence comparisons, and presence and location of clustered cysteines in specific plastid transporters of 2-oxoglutarate, to examine their evolution away from more generalized transporters. This transition would be to adopt the capability of internalizing 2-oxoglutarate alone or with superior specificities at the expense of malate. In phylogeny, the specific 2-oxoglutarate transporters (Cluster 1) are clustered in a separate clade away from 2 clades of general transporters (Cluster 2 and 3). The exclusivity (Cluster 1) and promiscuity of transporters (Cluster 2 and 3) compared to Arabidopsis counterparts characterized prior to this study, were used as a benchmark for my study. Within this mother clade of exclusive transporters, C4 and C3 2-oxoglutarate transporters once again form separate clusters of monophyly. Furthermore, a pattern of Cys -X-X-Cys-X(19)-Cys is conserved within the 2-oxo-glutarate only transporters that is missing in general transporters. Cysteines which are functionally key residues are inferred to be mediating intra- or interreactive disulfide bond formation or using a thiol (sulfhydryl) group for transport or to be forming a metal binding site. When a disulfide bond prediction tool was employed, it showed with negligible doubt that the Cys-X-X Cys-X(19) -Cys region was a strong contender for 2 separate disulfide bonds, although the middle cysteine was predicted to be involved in both. In addition, Cluster 2 general Zea mays $\mathrm{C} 4$ transporters are shown to be more recalcitrant to mutations of cysteines, compared to Panicum and Oryza counterparts. The study of 2oxoglutarate and its availability in the chloroplast could play a two-prong role in $\mathrm{C} 4$ plants: to be a candidate for synthesis of bundle sheath cell Rubisco enzyme, which makes up $\sim 50 \%$ of plant proteins, via ammonia assimilation, and even playing a role in carbon-centered biochemical pathways. This study could greatly facilitate choices in the tinkering of the right transporters for a future $\mathrm{C} 4$ rice in a climate change impacted world.
\end{abstract}

Received: 31 Aug 2020

Accepted: 26 May 2021

\section{Key words:}

2-oxoglutarate

$\mathrm{C} 4$ rice

C4 photosynthesis

Cysteines

Malate

*Corresponding author: dilantha@sci.sjp.ac.lk

\section{Introduction}

There are two main global divisions in relation to flowering plants; dicot versus monocot and C3 against $\mathrm{C} 4$ photosynthesis. The former has been estimated to have taken place between 150-140 Mya, while the latter division is suggested to have taken place between 35-32 Mya (Chaw et al., 2004; Christin et al., 2008). The former can be corroborated by key anatomical features, and the latter has structural (Kranz anatomy, single cell C4 photosynthesis etc), physiological (less need of water) and biochemical (enzymes such as PEP

Carboxylase used as the first assimilation step in C4 photosynthesis) variations (Lundgren et al., 2014; Juric et al., 2017). C4 photosynthesis has a number of features that are more promising for survival in a rapidly heating world influenced by greenhouse

This is an open-access article distributed under the terms of the Creative Commons Attribution 4.0 International License, which permits unrestricted use, distribution and reproduction in any medium provided the original author and source are credited. 
gases and has independently evolved in at least 65 occasions, which consolidates the emergence and reemergence of a convergent evolutionary fate. With this in mind, there is a worldwide effort to transform the staple of Asia - the domesticated genus Oryza from a C3 engine into a supercharged $\mathrm{C} 4$ photosynthetic system where $\mathrm{CO}_{2}$ will be concentrated at the Rubisco (Ribulose 1-, 5Bisphosphate Carboxylase) active site (Zhu et al., 2010; Feldman et al., 2014).

One of the key molecules that is common to nitrogen assimilation and many carbon related phenomena is 2-oxoglutarate (Woo et al., 1987; Taniguchi et al., 2002). This molecule is synthesized as a product of the Krebs cycle and then is internalized into plastids for the concomitant transformation of its organic backbone into an amino acid, glutamate, using ammonia. C3 plants and C4 counterparts possess transporters for the transfer (exchange) of 2oxoglutarate with malate (for the internalization of the former), while there are more general counterparts which are less specific for the organic molecule 2-oxoglutarate (Taniguchi et al. 2002). 2oxoglutarate has influence on two major elements in plants - the canonical element in terms of macro nutrients, nitrogen, and the one used for autotrophy, the element carbon, which is converted to 3phosphoglycreate by Rubisco and used for organic building endeavors (Huergo \& Dixon, 2015). In nitrogen assimilation, 2-oxoglutarate is converted to glutamate by two enzymes, glutamate synthase (GOGAT) and glutamate dehydrogenase (GDH), the latter being able to synthesize a single glutamate and the former two glutamates respectively, starting from the 2-oxoglutarate substrate (Huergo \& Dixon, 2015). In plants, the synthesized glutamate donates an amide group to a glyoxylate, which results in the formation of glycine in photorespiratory pathway (Dellero et al., 2016).

Cysteines are thought to be one of the most functionally significant amino acids which transpired later in organismal evolution. A hallmark in cysteine function includes disulfide bond formation which gives a protein extra stability in three-dimensional structure. Cysteines possess thiol groups (the only amino acid to utilize such a group), are able to form disulfide bonds using two cysteine residues, are found as a highly conserved residue in protein sequences, forms clusters in close proximity, possess high metal binding affinities, while having two bases of interpretation on its hydrophobic/nonhydrophobic nature (Poole, 2015).

In a recent study, there was the demonstration by the $\mathrm{C} 4$ rice consortium that transgenic expression of key enzymes related to maize $\mathrm{C} 4$ photosynthesis inside the rice leaf, does not have a resulting increase in yield or vegetative growth parameters (biomass), despite correct localization in planta of the transgenic enzymes (Karki et al., preprint). The compartmentalization of photosynthesis and associated photorespiration, does make the membrane-bound transporters key contenders for the transport of small organic molecules, chiefly $\mathrm{C} 4$ compounds (Dellero et al., 2016). If at all C4 rice is to be a success, the biochemical currents should be manipulated with diligence, along the conduits between mesophyll and bundle sheath cell, cytosol and plastid and other compartments, as too longerrange cell wall (apoplastic transport) and plasmodesmata (symplastic transport) based transport. There are many protein gatekeepers on border control at membranes (Taniguchi et al., 2002) and such proteins have to be taken into perspective, if we are to build a C4 prototype. Furthermore, there is the requirement of looking at the enigmatic 2-oxoglutarate, of which the key functions are all not known in the present (Huergo \& Dixon, 2015). How 2-oxoglutarate can be sensed and used in $\mathrm{C}$ and $\mathrm{N}$ biochemistry can be the holy grail in relation to a future GM-based $\mathrm{C} 4$ rice crop.

Finally, there are two kinds of 2-oxoglutarate shuttles; the specific one and the common one, or simply the specialist and the generalist (Taniguchi et al., 2002). While the specific one - albeit associated with 2-oxoglutarate only - is now known to have two biochemical shuttle capacities; oxaloacetate/malate and 2-oxoglutarate/malate (Kinoshita et al., 2011). The former or oxaloacetate/malate shuttles are known as the malate valves (Selinski \& Scheibe, 2019) and are key due to the non-permeability of $\mathrm{NADH}$ and NADPH across membranes, along with the enzymes, malate dehydrogenases.

This paper is an exploration into what makes the specialist 2-oxoglutarate transporters different from generalist counterparts. Observations on phylogeny, sequence divergence, amino acid composition and reactivities between unique cysteines are inferred here to play key roles in making such transporters unique.

\section{Materials and Methods}

\subsection{Secondary Structure Prediction}

The secondary structure prediction service PSIPRED 4.0 (http://bioinf.cs.ucl.ac.uk/psipred/) was used to showcase the helixes, beta strands, and coils (McGuffin et al., 2000).

\subsection{Phylogenetic Reconstructions}

The non-redundant downloaded amino acid sequences (as FASTA files) from each query were first aligned with the ClustalW algorithm using MEGA version X (default parameters) (Sohpal et al., 2010) which were converted to the MEGA 
sequence format, and phylogenetic reconstruction was performed using the neighborhood joining/maximum parsimony methods with support from 500 bootstrap replications. There was no assignment of outgroups

\subsection{Prediction of disulfide bonds}

The protein >XP_015620646.1 (a specific 2oxoglutarate transporter) from the genus Oryza was searched against the DiANNA server (http://clavius.bc.edu/ clotelab/DiANNA/) for the identification of likely disulfide bond pairs.

\subsection{Multiple sequence alignments}

The non-redundant downloaded amino acid sequences (as FASTA files) were used for sequence alignment using the ClustalW algorithm using MEGA version X (default parameters) (Sohpal et al., 2010).

\section{Results and Discussion}

2-oxoglutarate transporters from the genus Oryza (C3) and the genus Panicum (those only made of C4 species) including those that were both general and specific for 2-oxoglutarate, from the NCBI protein sequences database, were first downloaded. The threshold value was set as 30\% identity (Structural Classification Of Proteins (SCOP) defined levels) and $30 \%$ coverage (arbitrary value) to zoom in on authentic candidates and away from false positives (Lo Conte et al., 2000). For both C3 rice and C4 Panicum, there were three clear clusters of dicarboxylate transporters, which showed that there were three evolutionary outcomes in both $\mathrm{C} 3$ and $\mathrm{C} 4$ candidate plants (Figure 1).

In a study performed in 2002, a recombinant AtpOmt1 protein from Arabidopsis thaliana, produced using a yeast expression system, was able to transport "exclusively" 2-oxoglutarate, while the related AptDct1 protein was able to transport both 2oxoglutarate with glutamate suggesting the latter was a general transporter (Taniguchi et al., 2002). However, this is now disputed due to the capability of AptOmt1 to internalize oxaloacetate into the chloroplast at the expense of malate (Kinoshita et al., 2011). The Arabidopsis AtpOmt1 protein was found as a divergent node in phylogeny that deviated prior to the emergence of $\mathrm{C} 4$ photosynthesis and clustered with 6 Oryza (C3), 3 Zea (C4) and 4 Panicum (C4) proteins as a monophyletic clade (Figure 2). This monophyletic clade composed of the "exclusive" 2-oxoglutarate transporters was named Cluster 1. The AtpOmt1 protein would have been present at a time predating the emergence of $\mathrm{C} 4$ photosynthesis, and would have diverged down the dicot lineage after the breakaway of monocots from the prior common plant lineage dating back to before 140-150 Mya.

On the other hand, the AtDct1 protein was found as a basal node to two other Clusters (Cluster 2 and 3), which demonstrates that the emergence of the two Clusters postdated the dicot-monocot breakaway (Figure 2). From sequence similarity and phylogeny, it is stated that the AtDct1 protein and the remaining proteins from Cluster 2 and Cluster 3 are all general dicarboxylate transporters, using a combination of sequence similarity, with monophyly. The general transporters will likely show promiscuity to accommodate myriad compounds to be transported across as cargo to the plastid interior, while letting malate be transported out to the cytosol. It is though interesting that once the monocot clade emerged from the monocot-dicot common lineage, there too was an extra divergence event which made way to the formation of Cluster 2 and Cluster 3 as separate Clusters, which predates the 35 Mya emergence/breakaway of the $\mathrm{C} 4$ photosynthesis group (Figure 2). Basically, following the breakaway which took place between $\sim 140$ and $\sim 35$ Mya, there would have been a bifurcation, to let two Clusters - 2 and 3 - emerge from the common monocot lineage.

Sequence features that were different between the exclusive 2-oxoglutarate (Cluster 1) and the generalized transporters (Cluster 2 and 3) were scrutinized. When sequence alignments were produced and analyzed, there were three cysteines, clustered close to each other that were only present in Cluster 1 (in both $\mathrm{C} 3$ and $\mathrm{C} 4$ candidates) but were missing in general transporter clusters. The distance between the first and the second cysteine was 2 residues and the space between the second and the third cysteines was 19 residues (Figure 3 ). The motif was named as Cys -X-X-Cys-X(19)-Cys. The same three cysteines too are conserved in the Arabidopsis AtpOmt1 protein CVACGSNVGDGTEHRLGSWLMLTC-) suggesting that for specialist 2-oxoglutarate transporters, there is no difference in relation to the monocot-dicot divergence or the emergence of $\mathrm{C} 3 / \mathrm{C} 4$ photosynthesis systems, indicating events in convergent evolution. However, cysteines are relatively rare in transmembrane proteins which infer that the three-cysteine region may be crucially significant for a specific function. 


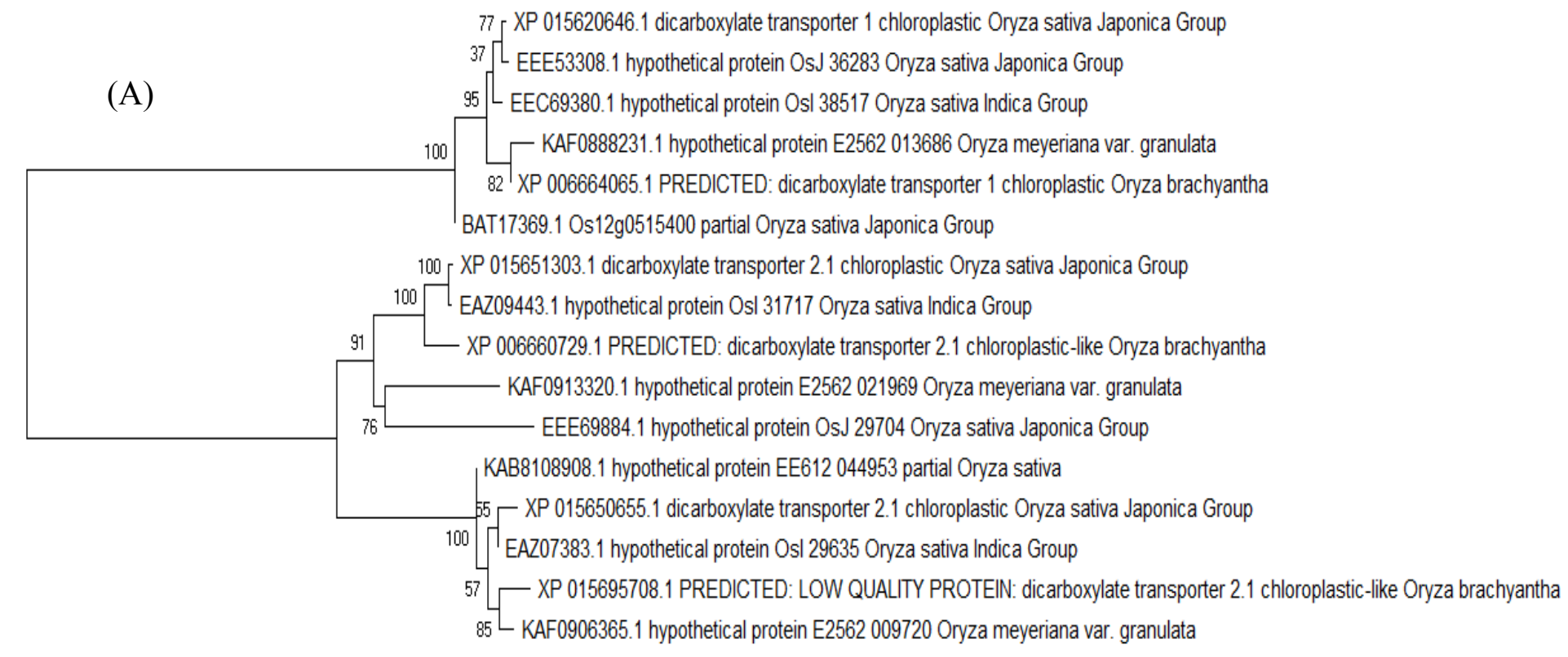

0.10

(B)

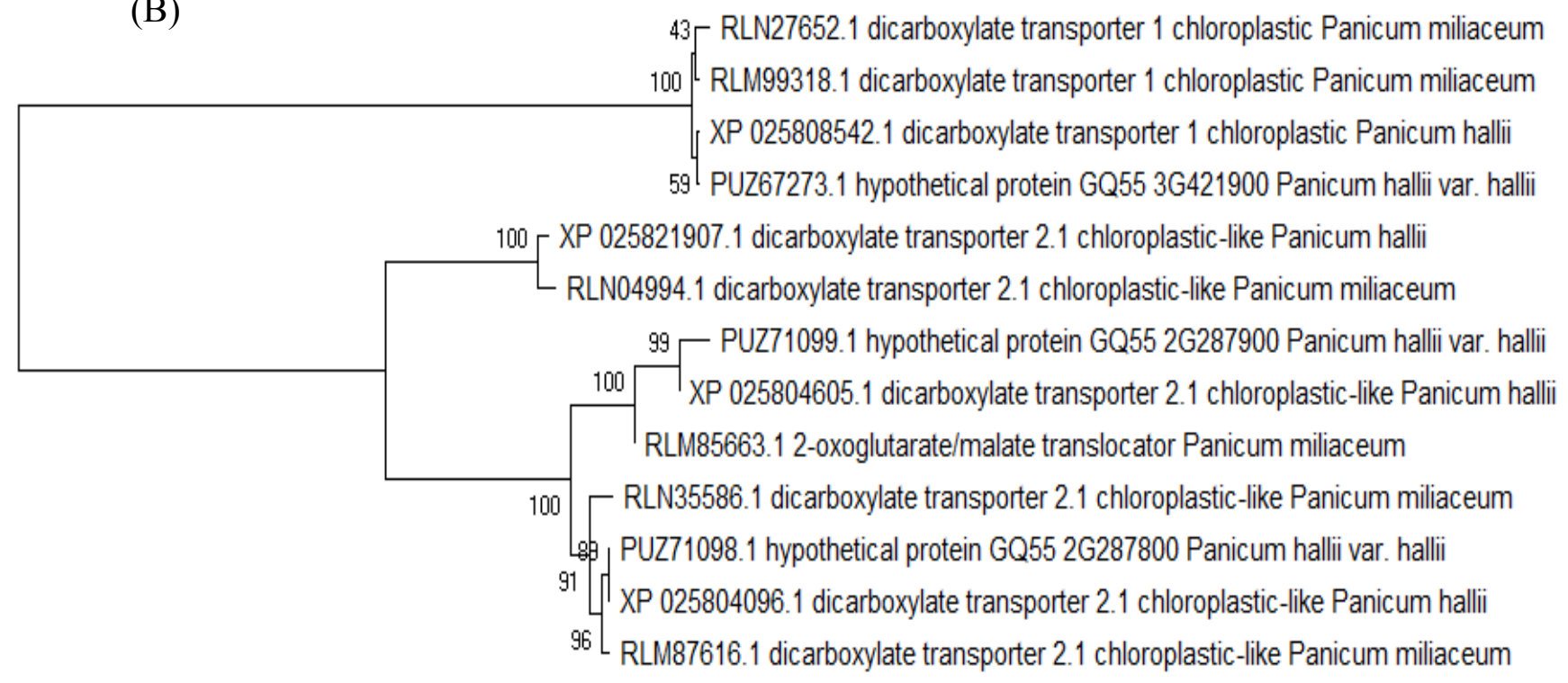

0.10

Figure 1(A) The amino acid sequences comprising diarboxylate $(\mathrm{C} 4,2$-oxoglutarate) transporters from the genus Oryza were first aligned using the ClustalW algorithm using MEGA version X and the phylogenetic reconstruction performed using the Neighborhood Joining method with support from 500 bootstrap replications.

Figure 1(B) The amino acid sequences of dicarboxylate (C4, 2-oxoglutarate) transporters from C4 Panicum species were first aligned using the ClustalW algorithm using MEGA version $X$ and the phylogenetic reconstruction performed using the Neighborhood Joining method with support from 500 bootstrap replications. 


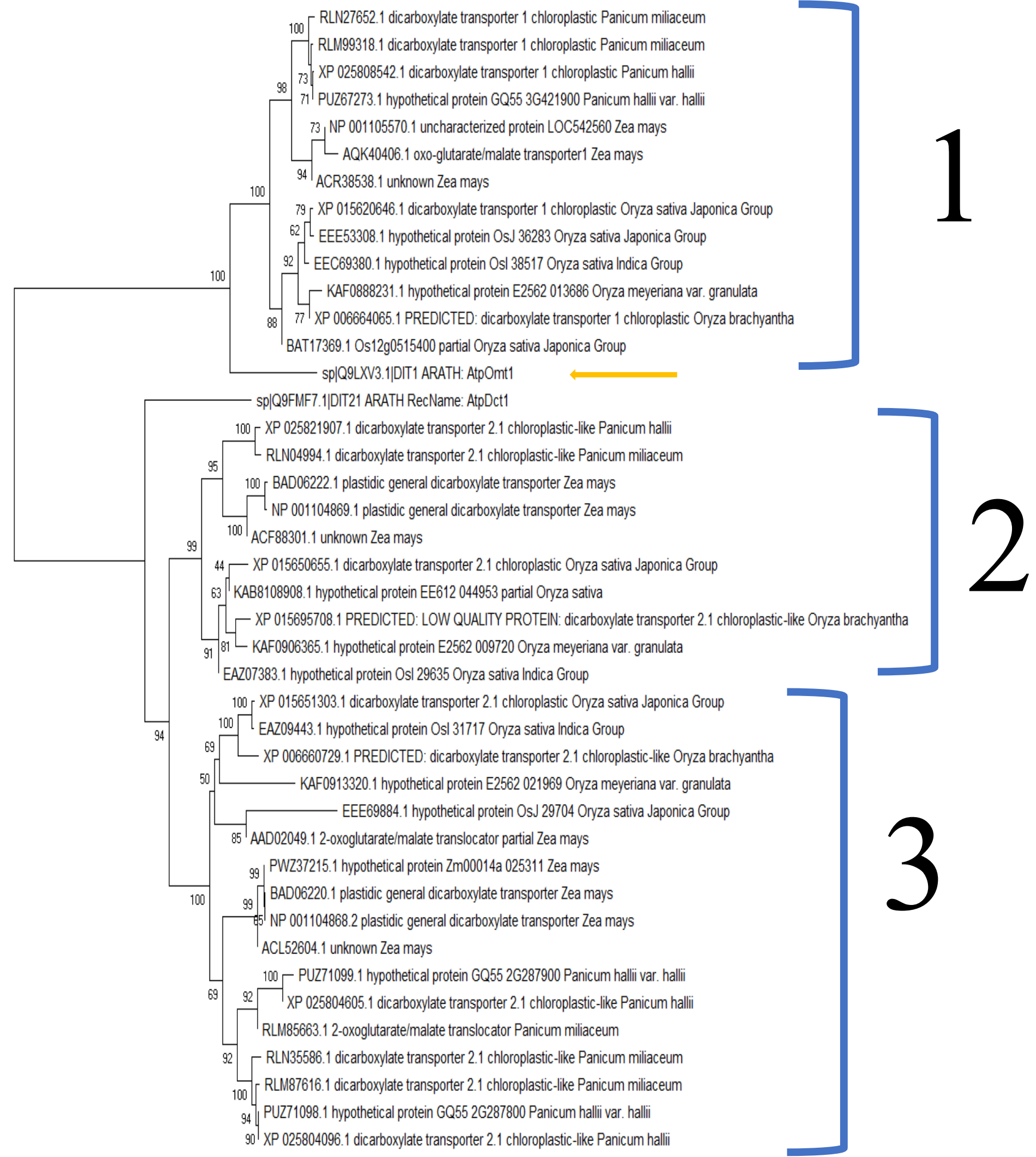

Figure 2: The amino acid sequences (42 in number) comprising dicarboxylate (2-oxoglutarate) transporter proteins from the genera Oryza, Panicum and Zea with two Arabidopsis proteins (AtpOmt1 and AtpDct1), were first aligned using the ClustalW algorithm using MEGA version $\mathrm{X}$ and the phylogenetic reconstruction performed using the Neighborhood Joining method with support from 500 bootstrap replications. The identities of the nodes are present at the nodal tips. Cluster 1, Cluster 2 and Cluster 3 are shown in brackets. 
There are three possible biochemical fates to the above string (three) of cysteines. First, they can form intra-molecular disulfide bonds, or if the arrangement of the monomers is as dimers or multimers, then there is the possibility of intermolecular disulfide bond formation. When the secondary structure of the Oryza protein (>XP_015620646.1) was analyzed using bioinformatics (McGuffin et al. 2000), The first two cysteines (Cys X-X Cys) were found in an extracellular domain and the third cysteine was found in a region making interactions with the plastid membrane, perhaps in a helical disposition (Figure 4). The Cys-X-X-Cys region is one of the most commonly found motifs in proteins, although this motif is found less in plants compared to most other complex as well as basic life forms (Miseta and Csutora 2000). It is suggested that either of these three cysteines could form intra-molecular disulfide bonds. To further explore this possibility, the Oryza sativa japonica sequence >XP_015620646.1 (a specific 2-oxoglutarate transporter belonging to Cluster 1) was used against the DiANNA server (http://clavius.bc.edu/ clotelab/DiANNA/) for the identification of likely disulfide bond pairs (Ferre \& Clote, 2005; Ferre \& Clote, 2006). Three cysteines (Cys -X-X-Cys-X(19)-Cys) was found as being reactive in disulfide bonds in the following combinations. Disulfide bonds 232-235 (Cysteine 1 and Cysteine 2) or 235-255 (Cysteine 2 and Cysteine 3 ) were identified by DiANNA as the likely sources of disulfide bonds (Table 1). The fact that the second cysteine is found in both bonds, attests to a likely scenario that only one of the above bonds are present at any given time.

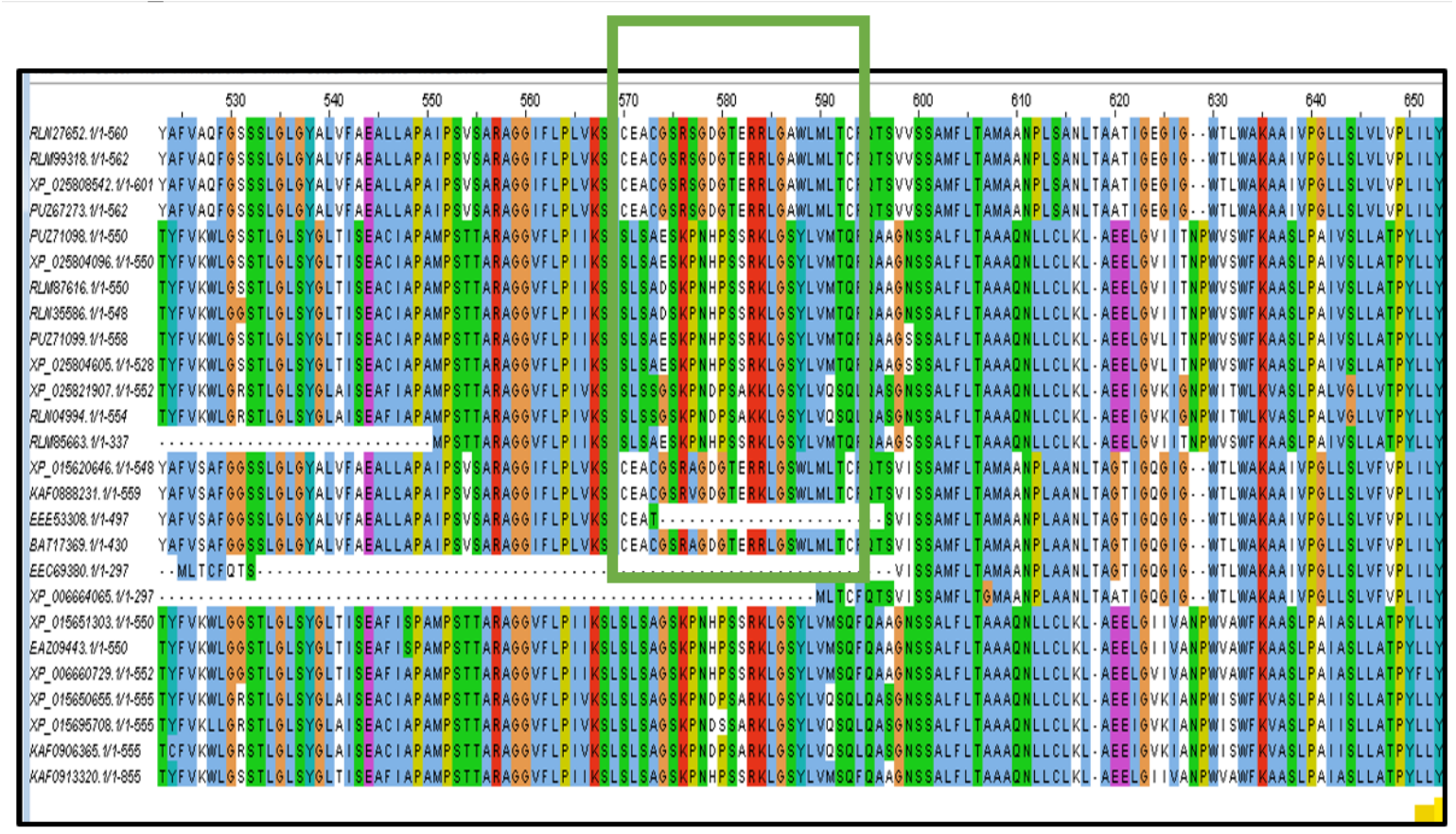

Order of Cysteines $=$ Cys-X-X- Cys X(19) Cys

Figure 3: The sequence alignment of $\mathrm{C} 3$ and $\mathrm{C} 4$ dicarboxylate (2-oxoglutarate) transporters from the genus Oryza and the genus Panicum. The three cysteines containing region is shown in the sequence alignment and the exact sequence pattern is shown below in a size 20 font. 


\begin{tabular}{llll}
\multicolumn{1}{|l}{ Strand } & Helix & Coil & $\square$ Disordered \\
$\square$ Disordered, protein binding & Putative Domain Boundary & Membrane Interaction & Transmembrane Helix \\
\hdashline Extracellular & Re-entrant Helix & Cytoplasmic & Signal Peptide
\end{tabular}

Figure 4: Output from the secondary structure prediction tool PSIPRED 4.0 for Oryza sativa Japonica sequence $>$ XP_015620646.1 showing membrane interacting regions, beta strands and loops/coils

Table 1: Predicted disulfide bonds and their probabilities based on scores, when the Oryza sativa Japonica sequence $>$ XP_015620646.1 was searched using the DiANNA server (http://clavius.bc.edu/ clotelab/DiANNA/). The most likely scores respectively, involve the regions demarcated by the Cys- X-X -Cys X(19) Cys and are a reflection of their candidacy for being reactive as disulfide bonds.

\begin{tabular}{|c|c|l|l|}
\hline Cysteine sequence position & Distance & \multicolumn{1}{|c|}{ Bond } & Score \\
\hline $14-232$ & 218 & TAPLTCHHLGL-LVKSLCEACGS & 0.01037 \\
\hline $14-235$ & 221 & TAPLTCHHLGL-SLCEACGSRAG & 0.01038 \\
\hline $14-255$ & 241 & TAPLTCHHLGL-WLMLTCFQTSV & 0.01038 \\
\hline $14-390$ & 376 & TAPLTCHHLGL-VTWKECLGEAV & 0.01037 \\
\hline $232-235$ & 3 & LVKSLCEACGS-SLCEACGSRAG & 0.99775 \\
\hline $232-255$ & 23 & LVKSLCEACGS-WLMLTCFQTSV & 0.01072 \\
\hline $232-390$ & 158 & LVKSLCEACGS-VTWKECLGEAV & 0.03247 \\
\hline $235-255$ & 20 & SLCEACGSRAG-WLMLTCFQTSV & 0.97288 \\
\hline $235-390$ & 155 & SLCEACGSRAG-VTWKECLGEAV & 0.01482 \\
\hline $255-390$ & 135 & WLMLTCFQTSV-VTWKECLGEAV & 0.01365 \\
\hline
\end{tabular}


The DiANNA server (Ferre and Clote, 2005, 2006) uses a five step process for the identification of disulfide bonds: (1) PSIPRED is used for the determination of secondary structural motifs; (2) PSI-Blast is used against the SWISS-PROT database to obtain a multiple sequence alignment (3) (1) and (2) together lead a neural network mechanism for training purposes of the prediction methodology (4) The oxidation states of the cysteines are predicted and finally (5) Rothberg's usage of Gabow's maximum weighted matching parameters are used for the clear demarcation of likely disulfide bond formations (Ferre \& Clote, 2006).

On the other hand, though, there have been instances where transmembrane cysteines have facilitated the transport of compounds to the inside of a compartment, which points to any of the cysteines, being structurally partial to the permeation of the membrane by the organic molecule, 2-oxoglutarate (Jimenez-Vidal et al., 2004). Since the chloroplast is widely an oxygen rich environment (due to photosynthesis), it is likely that disulfide bond formation would be preferred. Yet another possibility is that the sulfhydryl groups are acting as sensors of 2-oxoglutarate or to bind to metal ions, thereby enabling a more discrete function.

A preserved cysteine is found in Cluster 2 of Zea mays general $\mathrm{C} 4$ transporters but are missing in counterparts of the same clade that are Panicum or Oryza in identity (Figure 5 and 6). Even in the basal AtDct1 protein there is a cysteine to glycine mutation indicating that the Zea mays sequences may have preserved the cysteine function while the near and far counterparts in Cluster 2, may have lost the putative functional cysteine to a glycine (Figures 5 and 6). It would be interesting to transfer the candidate Zea mays sequences in Cluster 2 to the $\mathrm{C} 3$ rice plant to ascertain their true contribution to the cellular transport of $\mathrm{C} 4$ compounds. It is inferred that the cysteine to glycine mutation to be a loss of function mutation. The cysteine to glycine mutation according to the genetic code is not a simple wobble (third) position mutation, and this consolidates my theory that this is likely a functional strategy preserved in Zea mays, that is common to all $\mathrm{C} 4$ transporters of this food crop.

There is the need to explore the role of the three cysteines using site-directed mutagenesis or CRISPR/Cas9 and perhaps even using the Xenopus oocyte expression system to study the transporter functions. It is crucial to learn how 2-oxoglutarate is channeled across the many conduits that make up compartmentalized anatomies and substructures (example - Figure 7). With the absence of a clear tertiary structure, it is difficult to identify any other residue that may have a role to play in the biology of 2-oxoglutarate transporters. The rather unique syndrome of specific 2-oxoglutarate transporters is a sound convergent adaptation, and genetically incorporating such a transporter in $\mathrm{C} 4$ rice should be a biochemical bonanza for the rice scientists working on $\mathrm{C} 4$ rice. 

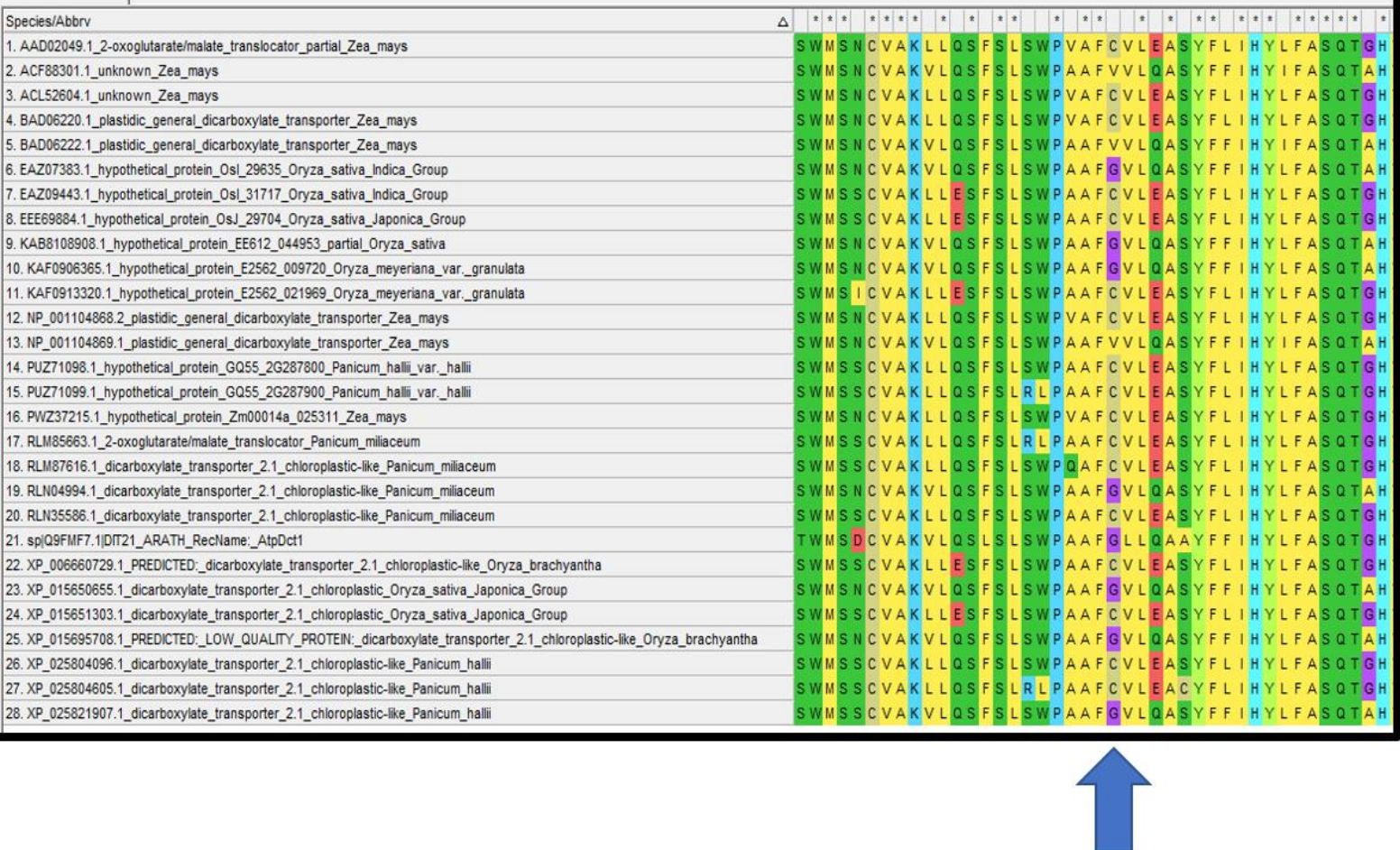

Figure 5: The sequence alignment of all 28 sequences representing Cluster 2 and Cluster 3, showcasing the key cysteine $\rightarrow$ glycine mutation that is only found in Oryza and Panicum general C4 transporters of Cluster 2 but are missing in Zea mays transporters, suggesting a likely preserved cysteine implying functional significance. 


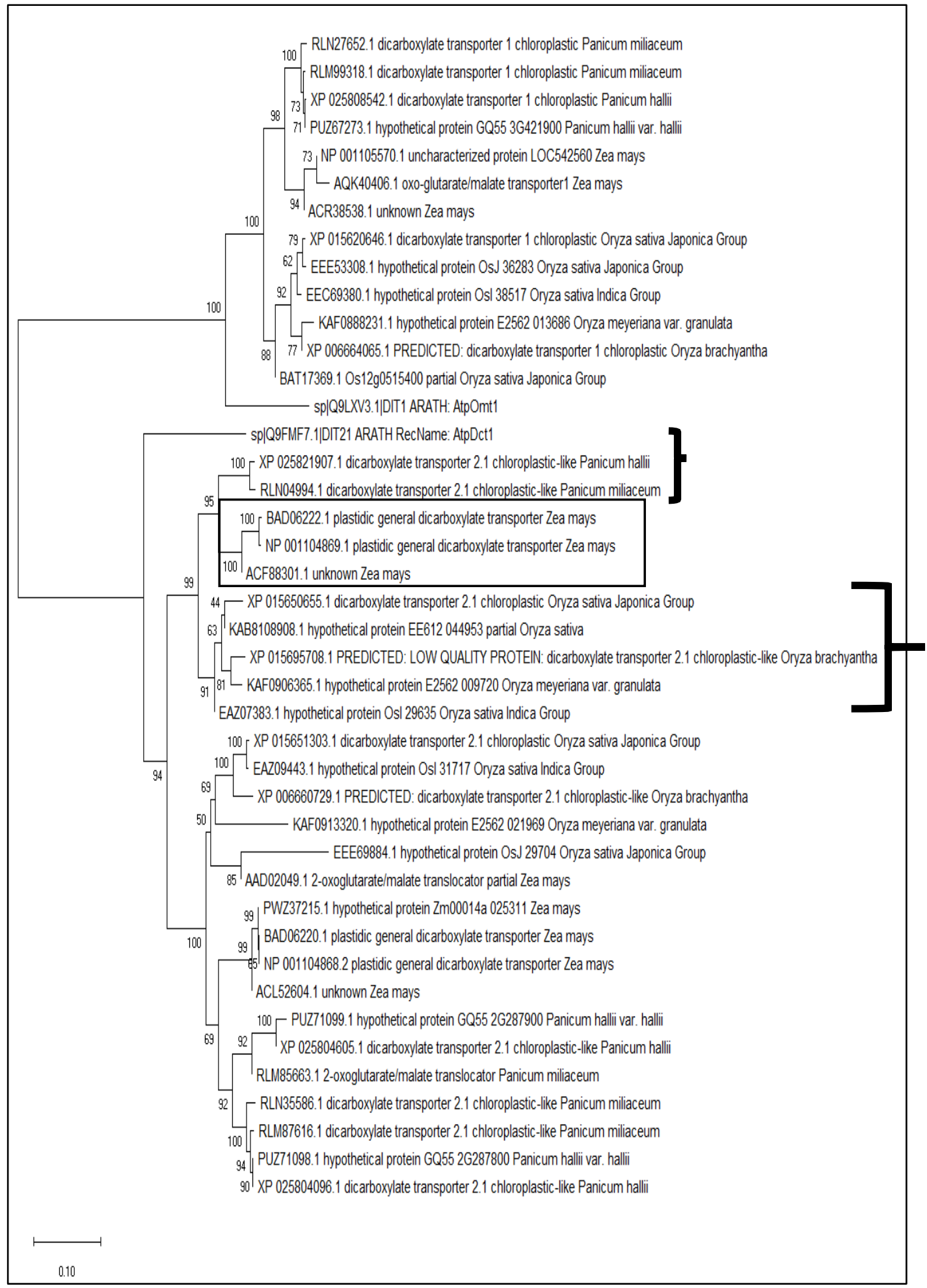

Figure 6: The amino acid sequences ( 42 in number) comprising dicarboxylate (2-oxoglutarate) transporter proteins from the genera Oryza, Panicum and Zea with two Arabidopsis proteins (AtpOmt1 and AtpDct1) were first aligned using the ClustalW algorithm using MEGA version $\mathrm{X}$ and the phylogenetic reconstruction performed using the Neighborhood Joining method with support from 500 bootstrap replications. The Cluster 2 Zea mays proteins that have an extra cysteine are demarcated in an orange box while the Cys $\rightarrow$ Gly mutations that are common to both Panicum and Oryza sequences of Cluster 2 are shown in blue brackets. 


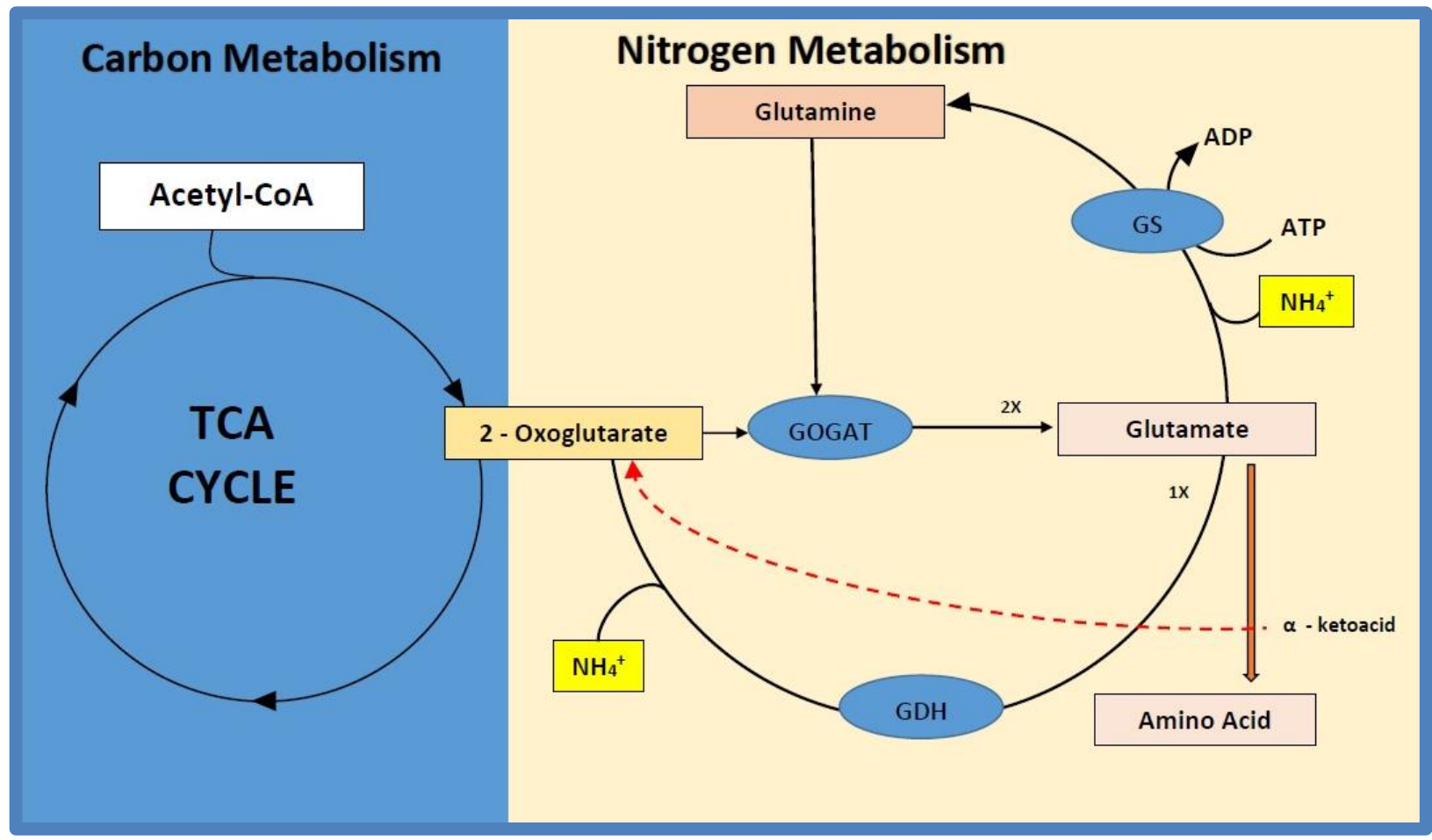

Figure 7: The transport and subsequent biochemical changes performed on the 2-oxoglutarate molecules in the chloroplast.

GOGAT - Glutamine oxoglutarate aminotransferase; GDH - Glutamate dehydrogenase; GS - Glutamine Synthetase.

\section{Conclusions}

It is showcased here the founding diversity in $\mathrm{C} 4$ transporters capable of transport of 2-oxoglutarate to the inside of the plastid, while focusing on their divergence from general transporters incorporating cysteines in their extracellular or membraneinteracting helical domains. The phylogeny of unique 2-oxoglutarate only transporters suggests that $\mathrm{C} 3$ and $\mathrm{C} 4$ photosynthesis-based divisions share the same cysteine architecture perhaps alluding to common shared functionality. The Arabidopsis AtOmt1 protein too shares with this monophyletic clade - although diverging at the dicot-monocot bifurcation - the three cysteines (Cys -X-X-Cys$\mathrm{X}(19)-$-Cys) architecture. It is also shown that the presence of a conserved cysteine in all Cluster 22 oxoglutarate transporters of Zea mays, which is transformed to a glycine in non-Zea proteomes, which we infer to be a loss of function mutation. In all, learning the architecture of cysteines and their possible contributions to transport of 2-oxoglutarate should be a valuable addition to the strategic tinkering of transporters inside a future $\mathrm{C} 4$ rice.

\section{Acknowledgement}

I acknowledge Michelle Alexander whose great artistic effort yielded figure 7 .

\section{References}

Chaw, S. M., Chang, C.C., Chen, H.L. and Li, W.H. (2004) Dating the monocot-dicot divergence and the origin of core eudicots using whole chloroplast genomes. Journal of Molecular Evolution 58(4): 424-441.

Christin, P. A., Besnard, G., Samaritani, E., Duvall, M.R., Hodkinson, T.R., Savolainen, V., and Salamin, N. (2008) Oligocene CO2 decline promoted $\mathrm{C} 4$ photosynthesis in grasses. Current Biology 18(1): 37-43.

Dellero, Y., Jossier, M., Schmitz, M., Maurino V.G., Hodges, W. (2016) Photorespiratory glycolateglyoxylate metabolism, Journal of Experimental Botany 67(10):30413052, https://doi.org/10.1093/jxb/erw090

Feldman, A. B., Murchie, E.H., Leung, H., Baraoidan, M., Coe, R., Yu, S.M., Lo, S.F. and Quick, W.P. (2014) Increasing leaf vein density 
by mutagenesis: laying the foundations for $\mathrm{C} 4$ rice. PLoS One 9(4): e94947.

Ferre, F. and Clote, P. (2005) DiANNA: a web server for disulfide connectivity prediction. Nucleic Acids Research 33(Web Server issue): W230-232.

Ferre, F. and Clote, P. (2006) DiANNA 1.1: an extension of the DiANNA web server for ternary cysteine classification. Nucleic Acids Research 34(Web Server issue): W182-185.

Huergo, L.F., Dixon R. (2015) The Emergence of 2Oxoglutarate as a Master Regulator Metabolite. Microbiology and Molecular Biology Reviews 79: 419-35. doi:10.1128/MMBR.0003815

Jimenez-Vidal, M., Gasol, E., Zorzano, A., Nunes, V., Palacin, M., and Chillaron, J. (2004) Thiol modification of cysteine 327 in the eighth transmembrane domain of the light subunit $\mathrm{xCT}$ of the heteromeric cystine/glutamate antiporter suggests close proximity to the substrate binding site/permeation pathway. Journal of Biological Chemistry 279(12): 11214-11221.

Juric, I., Gonzalez-Perez, V., Hibberd, J.M., Edwards, G., and Burroughs, N.J. (2017).Size matters for single-cell C4 photosynthesis in Bienertia. Journal of Experimental Botany $\mathbf{6 8}(2)$ : 255-267.

Kinoshita, H., Nagasaki, J., Yoshikawa, N., Yamamoto, A., Takito, S., Kawasaki, M., Sugiyama, T., Miyake, H., Weber, A.P.M. and Taniguchi, M. (2011). The chloroplastic 2oxoglutarate/malate transporter has dual function as the malate valve and in carbon/nitrogen metabolism. Plant Journal 65(1): 15-26.

Lo Conte, L., Ailey, B., Hubbard, T.J., Brenner, S.E., Murzin, A.G. and Chothia, C. (2000) SCOP: a structural classification of proteins database. Nucleic Acids Research 28(1): 257-259.

Lundgren, M. R., Osborne, C.P. and Christin, P.A. (2014) Deconstructing Kranz anatomy to understand C4 evolution. Journal of Experimental Botany 65(13): 3357-3369.

McGuffin, L. J., Bryson, K. and Jones, D.T. (2000) The PSIPRED protein structure prediction server. Bioinformatics 16(4): 404-405.

Miseta, A. and Csutora, P. (2000) Relationship between the occurrence of cysteine in proteins and the complexity of organisms. Molecular Biology and Evolution 17(8): 1232-1239.

Poole, L. B. (2015) The basics of thiols and cysteines in redox biology and chemistry. Free Radical Biology and Medicine 80: 148-157.

Selinski, J. and Scheibe, R. (2019) Malate valves: old shuttles with new perspectives. Plant biology (Stuttgart, Germany), 21 Suppl 1(Suppl Suppl 1), 21-30. https://doi.org/10.1111/plb.12869

Sohpal, V. K., Dey A., and Singh A. (2010) MEGA biocentric software for sequence and phylogenetic analysis: a review. International Journal of Bioinformatics Research and Applications 6(3): 230-240.

Taniguchi, M., Taniguchi, Y., Kawasaki, M., Takeda, S. Kato, T., Sato, S., Tabata, S., Miyake, H. and Sugiyama, T. (2002) Identifying and characterizing plastidic 2-oxoglutarate/malate and dicarboxylate transporters in Arabidopsis thaliana. Plant Cell Physiology 43(7): 706-717.

Woo, K. C., Flugge, U.I. and Heldt, H.W. (1987) A Two-Translocator Model for the Transport of 2Oxoglutarate and Glutamate in Chloroplasts during Ammonia Assimilation in the Light. Plant Physiology 84(3): 624-632.

Zhu, X. G., Shan, L., Wang, Y. and Quick, W.P. (2010) C4 rice - an ideal arena for systems biology research. Journal of Integrative Plant Biology 52(8): 762-770. 